\title{
Exponential stability of singularly perturbed systems with mixed impulses
}

\author{
Wu Yang ${ }^{\mathrm{a}, \mathrm{b}}$, Yan-Wu Wang ${ }^{\mathrm{a}, *}$, Irinel-Constantin Morărescuc ${ }^{\mathrm{c}}$, Jamal Daafouz $^{\mathrm{c}}$ \\ ${ }^{a}$ Key Laboratory of Image Processing and Intelligent Control, School of Artificial Intelligence and Automation, \\ Huazhong University of Science and Technology, Wuhan, 430074, China \\ ${ }^{b}$ School of Electrical Engineering, Guangxi University, Nanning, 530004, China \\ ${ }^{c}$ Université de Lorraine, CNRS, CRAN, F54000 Nancy, France
}

\begin{abstract}
This paper investigates the exponential stability problem for a class of singularly perturbed impulsive systems in which the flow dynamics is unstable and is affected at discrete time instants by impulses that have both destabilizing and stabilizing effects. More precisely the impulses have stabilizing effects on the slow variables but destabilizing effects on the fast ones. Thus, a first contribution of our work is related to stability analysis of singularly perturbed impulsive systems in the case when neither the flow dynamics nor the impulsive one is stable. In order to take full advantage of the jump matrix structure and its stabilizing effects on the slow dynamics, we introduce a new impulse-dependent vector Lyapunov function. This function allows us to better describe the behaviour between two consecutive impulses as well as the jumps at impulse instants. Several numerically tractable criteria for stability of singularly perturbed impulsive systems are established based on vector comparison principle. Additionally, upper bounds on the singular perturbation parameter are derived. Finally, the validity of our results is verified by two numerical examples.
\end{abstract}

Keywords: Singularly perturbed system, Mixed impulse, Exponential stability, Vector Lyapunov function

\section{Introduction}

Systems characterized by processes evolving on different time-scales appear in many fields of science and engineering: electric circuit, steel production, flexible joint robots or neural networks $[1,2,3,4]$. The mathematical formalism used to model these dynamics is provided by the singular perturbation theory. Usually one considers singularly perturbed systems (SPSs) involving two time-scales and though having two types of state variables: fast and slow. A small positive parameter $\varepsilon$ is used to describe the gap between the variation speed of the two types of variables. In the past decades, many achievements have been made on the analysis and synthesis of SPSs (see $[5,6,7,8,9,10,11]$ and the references therein).

\footnotetext{
*Corresponding author

Email address: wangyw@hust.edu.cn (Yan-Wu Wang )
} 
When SPSs are affected by impulses occurring at some discrete time we end up with a special class of dynamics called singularly perturbed impulsive systems (SPISs) [12, 13, 14]. Generally, the analysis and control synthesis of SPISs are complicated due to particular phenomena arising from the coexistence of multiple time-scales and the impulses. Nevertheless, there already exist in the literature few results on the analysis of SPISs. In [15, 16], Simeonov and Bainov established an exponential stability criteria of SPISs based on a vector Lyapunov function. The proposed method can efficiently use the different decay rates of the slow and fast variables to compensate the effects of the impulsive perturbation. Following the same idea, Alwan and Liu [17] extended these results to singularly perturbed switched systems with time delay and impulses. Chen et al [18] extended the results in $[15,16]$ to a class of singularly perturbed stochastic time delay systems with impulse effects. Recently, Ben Rejeb et al [19] proposed minimum dwell-time based stability conditions for a class of singularly perturbed hybrid systems exhibiting switches, impulses and changes of the slow/fast nature of the state variables. It's worth noting that in all these works both the reduced-order subsystem and boundary layer subsystem are assumed to be stable.

In the literature, from the impulsive magnitude point of view, the impulses are divided into three types: negative, positive and inactive [20, 21, 22, 23, 24, 25, 26]. More specifically, an impulse is said to be negative if it potentially destabilizes the dynamics, and to be positive if it has a stabilizing effect on the dynamics, otherwise it is called inactive. For example, in [27], Chen $e t$ al. considered the robust stability problem for SPISs with nonlinear perturbation. The obtained results in [27] show that the stability of the original systems can still be guaranteed provided that the impulses are stabilizing even if the flow dynamics is unstable. This result is further extended to a class of nonlinear singularly perturbed systems with delayed impulses in [28]. Note that the above-mentioned literature are devoted to investigating the positive or negative features of impulses separately. To study the positive and negative features of impulses within a unified framework, Lu et al. [20] introduced the so-called average impulsive interval and derived a unified synchronization criterion for directed impulsive dynamical networks. The proposed criterion is simultaneously effective for positive and negative impulses. Some recent results on synchronization of nonlinear dynamical networks with both synchronizing and desynchronizing impulses can be found in $[29,30,31]$. Precisely, the jump matrix is time varying and at some instants the impulses may be positive while at some other instants they are negative.

While the literature focuses mainly on the impact of stabilizing or destabilizing impulses on the overall system dynamics, in [32,33] the authors introduced and analyzed a class of systems with impulses having stabilizing (positive) effect on a part of the states and a destabilizing (negative) effect on the remaining states of the system. In the sequel these impulses will be called mixed impulses. To the best of our knowledge, the stability problem of SPSs with mixed impulses is still an open yet challenging issue, which motivates the research of this paper. We point out here that some achievements for synchronization of complex systems with mixed impulses in a single time scale are reported $[32,33]$. However, the proposed analysis methods cannot be directly used in our setting due to numerical ill-conditioning [10]. Besides, in our work, both the flow and jump dynamics of the considered systems are unstable, this renders challenges for estimating the bound of the divergent state, these technical difficulties make the classical results in $[32,33]$ inappropriate here. A supplementary challenge considered in this work is related to the computation of an upper bound on the singular perturbation parameter $\varepsilon$ guaranteeing the results can be applied.

The main objective of this paper is to provide exponential stability criteria for SPSs affected by mixed impulses. We emphasize that we are not focusing on the design of impulses or of the 
sequence of impulse instants, i.e., once the system is given we provide criteria to decide whether it is stable or not. It is worth noting that, unlike existing studies, our development considers the reduced order system is unstable. Our exponential stability criteria are established in term of linear matrix inequalities (LMIs) and they are based on the design of impulse-dependent vector Lyapunov function and the use of vector comparison principle. Moreover, the upper bound of the singular perturbation parameter is computed. Summing up, the contribution of this paper is twofold:

(1) We provide a stability analysis for a class of SPISs with mixed impulses in which the flow dynamics is unstable and the jump dynamics is stabilizing only the slow states.

(2) We develop a new tool for stability analysis of SPISs based on impulse-dependent vector Lyapunov function. This will allow us to better characterize the state evolution between two successive impulsive instants as well as the jump dynamics. Furthermore, using the vector comparison principle we derive some stability criteria, which are represented by a set of well-conditioned LMIs and can be checked easily. On top of that, we derive the upper bound of the singular perturbation parameter guaranteeing the results can be applied.

The rest of the paper is organized as follows. Section 2 gives the problem formulation and some preliminaries. Section 3 presents the exponential stability results for SPISs. Section 4 provides a numerical example. Section 5 draws some conclusions and proposes future works.

Notations: $\mathbb{R}, \mathbb{R}_{+}, \mathbb{R}^{n}$ and $\mathbb{R}_{+}^{n}$ denote the set of real numbers, the set of nonnegative real numbers, the set of the real $n$-dimensional vectors, and the set of the nonnegative real $n$-dimensional vectors, respectively. $\mathbb{N}$ and $\mathbb{N}^{+}$are the sets of nonnegative integer and positive integer, respectively. For a matrix $P$, the expression $P<0$ means that $P$ is real symmetric negative definite. We write $P \leq 0$ if all the components of the matrix $P$ are non-positive and we write $P \leq Q$ if $P-Q \leq 0$. We denote $\lambda_{\max }(A)$ and $\lambda_{\min }(A)$ the maximum and minimum eigenvalues of the matrix $A$, respectively. $\operatorname{He}\{A\}$ stands for $A+A^{T}$. The symbol $*$ within the matrix means the symmetric item in block matrices. The function $g(t, u): \mathbb{R}_{+} \times \mathbb{R}^{n} \mapsto \mathbb{R}^{n}$ is quasi-monotone nondecreasing if $u \leq v, u_{i}=v_{i}$ for some $i$ implies $g_{i}(t, u)<g_{i}(t, v)$. Finally, $\mathcal{V}_{0}$ is the set of functions $V: \mathbb{R}_{+} \times \mathbb{R}^{n} \mapsto R_{+}$satisfying the following properties (1) $\exists$ a sequence $\left(t_{k}\right)_{k \in \mathbb{N}}$ such that $V(t, x)$ is continuous on $\left[t_{k}, t_{k+1}\right) \times \mathbb{R}^{n}, \forall k \in \mathbb{N} ;(2)$ for each $x \in \mathbb{R}^{n}, \lim _{(t, y) \rightarrow\left(t_{k}^{+}, x\right)} V(t, y)=V\left(t_{k}^{+}, x\right)$; (3) $V(t, x)$ is locally Lipschitz in $x$.

\section{Problem Description}

Beside the problem description, this section presents some preliminaries including a numerical example illustrating the behavior of unstable SPS in presence of different types of impulses. This will further motivate the stability analysis in the next section.

Consider the following singularly perturbed impulsive systems:

$$
\begin{aligned}
& {\left[\begin{array}{c}
\dot{x}(t) \\
\varepsilon \dot{z}(t)
\end{array}\right]=\left[\begin{array}{ll}
A_{11} & A_{12} \\
A_{21} & A_{22}
\end{array}\right]\left[\begin{array}{l}
x(t) \\
z(t)
\end{array}\right], t \in\left[t_{k}, t_{k+1}\right),} \\
& {\left[\begin{array}{l}
x\left(t_{k}\right) \\
z\left(t_{k}\right)
\end{array}\right]=\left[\begin{array}{ll}
D_{11} & D_{12} \\
D_{21} & D_{22}
\end{array}\right]\left[\begin{array}{l}
x\left(t_{k}^{-}\right) \\
z\left(t_{k}^{-}\right)
\end{array}\right], k \in \mathbb{N},}
\end{aligned}
$$

where $x(t) \in \mathbb{R}^{n_{x}}$ is the slow state vector, $z(t) \in \mathbb{R}^{n_{z}}$ is the fast state vector, $\varepsilon$ is a small parameter that indicates the degree of the fast and slow dynamics separation. $A_{i j}, D_{i j}, i=1,2, j=1,2$ are 
constant real matrices with appropriate dimensions. As a standard hypothesis [10], assume that matrix $A_{22}$ is Hurwitz.

In this work we don't design the impulses time sequence but we assume the following properties hold true: (1) $0=t_{0}<t_{1}<\cdots<t_{k} \ldots$ is the monotonically increasing and unbounded sequence of impulse instants; (2) Denote $\tau_{k}=t_{k}-t_{k-1}, k \in \mathbb{N}^{+}$, then $0<\bar{\tau}_{1} \leq \tau_{k} \leq \bar{\tau}_{2}<\infty$, where $\bar{\tau}_{1}$ and $\bar{\tau}_{2}$ are positive constants.

In what follows, let $\mathcal{N}\left(\bar{\tau}_{1}, \bar{\tau}_{2}\right):=\left\{\left\{t_{j}\right\}_{j \in \mathbb{N}} ; 0<\bar{\tau}_{1} \leq \tau_{j} \leq \bar{\tau}_{2}<\infty, j \in \mathbb{N}^{+}\right\}$be the set of admissible impulsive time sequence.

Let $L=A_{22}^{-1} A_{21}$ and introduce the following change of variable:

$$
\left[\begin{array}{l}
x \\
z
\end{array}\right]=\left[\begin{array}{cc}
I_{n_{x}} & 0 \\
-L & I_{n_{z}}
\end{array}\right]\left[\begin{array}{l}
x \\
y
\end{array}\right],
$$

Using (3), system (1)-(2) in the variables $(x, z)$ becomes:

$$
\begin{aligned}
& {\left[\begin{array}{c}
\dot{x}(t) \\
\varepsilon \dot{y}(t)
\end{array}\right]=\left[\begin{array}{ll}
\bar{A}_{11} & \bar{A}_{12} \\
\bar{A}_{21} & \bar{A}_{22}
\end{array}\right]\left[\begin{array}{l}
x(t) \\
y(t)
\end{array}\right], t \in\left[t_{k}, t_{k+1}\right),} \\
& {\left[\begin{array}{l}
x\left(t_{k}\right) \\
y\left(t_{k}\right)
\end{array}\right]=\left[\begin{array}{ll}
\bar{D}_{11} & \bar{D}_{12} \\
\bar{D}_{21} & \bar{D}_{22}
\end{array}\right]\left[\begin{array}{l}
x\left(t_{k}^{-}\right) \\
y\left(t_{k}^{-}\right)
\end{array}\right], k \in \mathbb{N},}
\end{aligned}
$$

where $\bar{A}_{11}=A_{11}-A_{12} L, \bar{A}_{12}=A_{12}, \bar{A}_{21}=\varepsilon L \bar{A}_{11}, \bar{A}_{22}=A_{22}+\varepsilon L A_{12}, \bar{D}_{11}=D_{11}-D_{12} L, \bar{D}_{12}=$ $D_{12}, \bar{D}_{21}=L D_{11}+D_{21}-\left(L D_{12}+D_{22}\right) L, \bar{D}_{22}=L D_{12}+D_{22}$.

Although the system (4)-(5) looks similar to the original system (1)-(2), we highlight that the change of variables in (3) partially decouples the slow and fast states. Therefore, by using the change of variables in (3), we will be able to analyze the fast and slow state separately.

Assumption 1. $\bar{A}_{11}$ is not Hurwitz stable.

Remark 1. First we point out that we do not impose any restrictions on the matrix $A_{11}$ which can be either stable or unstable. Second, we notice that in $[15,16,17,18,19]$ both the reducedorder subsystem and boundary layer subsystem are assumed to be stable i.e., $\bar{A}_{11}$ is assumed to be Hurwitz stable. This means that the flow dynamic is stable based on the Tikhonov theorem [10] in absence of hybrid features, such as impulse. Notice that Assumption 1 places this study in a complementary case in which the flow dynamics of the reduced-order subsystem is unstable.

Remark 2. The impulses in $[15,16,17,18,19,27,28]$, are ether stabilizing or destabilizing. Motivated by the results in [32, 33], we analyze the behavior of (1)-(2) or (4)-(5) when the matrix $D$ defines mixed impulses. It is notable that the jump matrix D generating the impulses does not have to be Schur (stable) in order to get an overall SPIS which is stable.

Let us finish this section with the definition of the stability notion that we are trying to ensure.

Definition 1 ([27, 28]). For a given impulsive time sequence $\left\{t_{k}\right\}_{k \in \mathbb{N}} \in \mathcal{N}\left(\bar{\tau}_{1}, \bar{\tau}_{2}\right)$ and a given positive scalar $\varepsilon^{*}$, system (1)-(2) or (4)-(5) is said to be globally uniformly exponentially stable (GUES) for $\varepsilon \in\left(0, \varepsilon^{*}\right)$, if there exist positive constants $\varpi$ and $\lambda$ such that

$$
\|x(t)\|+\|y(t)\| \leq \varpi\left(\left\|x_{0}\right\|+\left\|y_{0}\right\|\right) e^{-\lambda\left(t-t_{0}\right)}
$$

for any $t \geq t_{0}$, any initial condition $\left(x_{0}, y_{0}\right)$ and any $\varepsilon \in\left(0, \varepsilon^{*}\right)$. Moreover, $\lambda$ is called the exponential convergence rate. 


\section{Stability Analysis}

In this section, the GUES conditions will be deduced for system (1)-(2) or (4)-(5). First, the GUES problem of system (1)-(2) or (4)-(5) is transformed into the stability of a two-dimensional vector comparison systems by employing an impulse-dependent vector Lyapunov function whose components are

$$
\begin{aligned}
V(t) & =x^{T}(t) P(t) x(t):=x^{T}(t)\left[(1-\rho(t)) P_{1}+\rho(t) P_{2}\right] x(t), \\
W(t) & =y^{T}(t) Q(t) y(t):=y^{T}(t)\left[(1-\rho(t)) Q_{1}+\rho(t) Q_{2}\right] y(t),
\end{aligned}
$$

where $P_{i}, Q_{i}(i=1,2)$ are positive definite matrices to be designed, and the function $\rho(t)$ is given as:

$$
\rho(t)=\frac{t_{k+1}-t}{t_{k+1}-t_{k}}, t \in\left[t_{k}, t_{k+1}\right), k \in \mathbb{N} .
$$

Then, by analyzing the properties of the considered two-dimensional vector comparison systems, we give the stability conditions for system (1)-(2) or (4)-(5). Moreover, the upper bound of the singular perturbation parameter is estimated by solving a one-dimensional optimization problem.

Remark 3. Lyapunov function (7)-(8) allows us to better capture the hybrid behaviour of the slow and fast states. Therefore, we can simultaneously compensate the divergence of the slow states during the flow and of the fast states during the jumps by the convergence of the slow state during jumps and of the fast state during the flow, respectively. It is also important to highlight that, for given impulsive time sequence $\left(t_{k}\right)_{k \geq 0}$, the proposed Lyapunov function (7)-(8) can be easily computed provided that $P_{i}, Q_{i}(i=1,2)$ are determined.

Now, we present an instrumental result allowing to transform the GUES problem of system (4)-(5) into the stability of a two-dimensional vector comparison systems by using (7)-(8).

Lemma 1. Consider the continuous-time impulsive system (1)-(2) or (4)-(5) and suppose that Assumption 1 holds. Let us also assume that the jump instant sequence $\left(t_{k}\right)_{k \geq 0}$ belongs to $\mathcal{N}\left(\bar{\tau}_{1}, \bar{\tau}_{2}\right)$ where $\bar{\tau}_{1} \leq \bar{\tau}_{2}$ are given positive scalars. If there exist positive definite matrices $P_{i}(i=1,2)$ and $Q_{i}(i=1,2)$, and some positive constants $\alpha_{i}(i=1,2), \beta_{j}(j=1,2,3), \gamma_{k}(k=1,2), \mu \in(0,1)$ and $v>1$ such that the following matrix inequalities hold

$$
\begin{aligned}
& \Phi^{1}(t) \triangleq\left[\begin{array}{cc}
\Omega^{1}(t) & P(t) A_{12} \\
* & -\alpha_{2} Q(t)
\end{array}\right]<0, \\
& \Phi^{2}(t) \triangleq \operatorname{He}\left\{Q(t) A_{22}\right\}+\beta_{1} Q(t)<0, \\
& \Phi^{3}(t) \triangleq\left[\begin{array}{cc}
-\beta_{3} P(t) & \left(L \bar{A}_{11}\right)^{T} Q(t) \\
* & \Omega^{2}(t)
\end{array}\right]<0, \forall t \in\left[t_{k}, t_{k+1}\right), k \in \mathbb{N}, \\
& \Psi_{1}=\left[\begin{array}{cc}
\bar{D}_{11}^{T} P_{2} \bar{D}_{11}-\mu P_{1} & \bar{D}_{11}^{T} P_{2} \bar{D}_{12} \\
* & \bar{D}_{12}^{T} P_{2} \bar{D}_{12}-\gamma_{1} Q_{1}
\end{array}\right] \leq 0, \\
& \Psi_{2}=\left[\begin{array}{cc}
\bar{D}_{21}^{T} Q_{2} \bar{D}_{21}-\gamma_{2} P_{1} & \bar{D}_{21}^{T} Q_{2} \bar{D}_{22} \\
* & \bar{D}_{22}^{T} Q_{2} \bar{D}_{22}-v Q_{1}
\end{array}\right] \leq 0,
\end{aligned}
$$


where $\Omega^{1}(t)=\operatorname{He}\left\{P(t) \bar{A}_{11}\right\}-\alpha_{1} P(t)+\frac{P_{1}-P_{2}}{\tau_{k+1}}, \Omega^{2}(t)=\operatorname{He}\left\{Q(t) L A_{12}\right\}-\beta_{2} Q(t)+\frac{Q_{1}-Q_{2}}{\tau_{k+1}}$. Then the following inequalities hold

$$
\begin{aligned}
& {\left[\begin{array}{c}
\dot{V}(t) \\
\dot{W}(t)
\end{array}\right] \leq C(\varepsilon)\left[\begin{array}{c}
V(t) \\
W(t)
\end{array}\right], t \in\left[t_{k}, t_{k+1}\right),} \\
& {\left[\begin{array}{c}
V(t) \\
W(t)
\end{array}\right] \leq J\left[\begin{array}{c}
V\left(t^{-}\right) \\
W\left(t^{-}\right)
\end{array}\right], t=t_{k}, k \in \mathbb{N} .}
\end{aligned}
$$

where $C(\varepsilon)=\left[\begin{array}{cc}\alpha_{1} & \alpha_{2} \\ \beta_{3} & \beta_{2}-\beta_{1} / \varepsilon\end{array}\right]$ and $J=\left[\begin{array}{cc}\mu & \gamma_{1} \\ \gamma_{2} & v\end{array}\right]$.

Proof. For $t \in\left[t_{k}, t_{k+1}\right), k \in \mathbb{N}$, the time derivatives of $V(t)$ and $W(t)$ along the trajectories of system (4)-(5) are given by

$$
\begin{aligned}
\dot{V}(t)= & 2 x^{T}(t) P(t) \dot{x}(t)+x^{T}(t) \dot{P}(t) x(t)=2 x^{T}(t) P(t)\left[\bar{A}_{11} x(t)+A_{12} y(t)\right]+\frac{1}{\tau_{k+1}} x^{T}(t)\left(P_{1}-P_{2}\right) x(t) \\
= & x^{T}(t)\left[\operatorname{He}\left\{P(t) \bar{A}_{11}\right\}+\frac{P_{1}-P_{2}}{\tau_{k+1}}-\alpha_{1} P(t)\right] x(t)+2 x^{T}(t) P(t) A_{12} y(t)-\alpha_{2} y^{T}(t) Q(t) y(t) \\
& +\alpha_{1} x^{T}(t) P(t) x(t)+\alpha_{2} y^{T}(t) Q(t) y(t)=\zeta^{T}(t) \Phi^{1}(t) \zeta(t)+\alpha_{1} V(t)+\alpha_{2} W(t)
\end{aligned}
$$

and

$$
\begin{aligned}
\dot{W}(t)= & \frac{2}{\varepsilon} y^{T}(t) Q(t) \varepsilon \dot{y}(t)+y^{T}(t) \dot{Q}(t) y(t) \\
= & \frac{2}{\varepsilon} y^{T}(t) Q(t)\left[\bar{A}_{21} x(t)+\bar{A}_{22} y(t)\right]+\frac{1}{\tau_{k+1}} y^{T}(t)\left(Q_{1}-Q_{2}\right) y(t) \\
= & \frac{1}{\varepsilon} y^{T}(t)\left[\operatorname{He}\left\{Q(t) A_{22}\right\}+\beta_{1} Q(t)\right] y(t)-\frac{1}{\varepsilon} y^{T}(t) \beta_{1} Q(t) y(t)-\beta_{3} x^{T}(t) P(t) x(t) \\
& +2 y^{T}(t) Q(t) L \bar{A}_{11} x(t)+y^{T}(t)\left[\operatorname{He}\left\{Q(t) L A_{12}\right\}+\frac{Q_{1}-Q_{2}}{\tau_{k+1}}-\beta_{2} Q(t)\right] y(t) \\
& +\beta_{2} y^{T}(t) Q(t) y(t)+\beta_{3} x^{T}(t) P(t) x(t) \\
= & \zeta^{T}(t) \Phi^{3}(t) \zeta(t)+\beta_{3} V(t)+\beta_{2} W(t)+\frac{1}{\varepsilon} y^{T}(t) \Phi^{2}(t) y(t)-\frac{\beta_{1}}{\varepsilon} W(t),
\end{aligned}
$$

where $\zeta^{T}(t)=\left[x^{T}(t) \quad y^{T}(t)\right]$.

Then, it can be deduced from conditions (9)-(11) that the inequality (14) holds.

For the impulsive instants, it follows from conditions (12) and (13) that

$$
\begin{aligned}
V\left(t_{k}\right) & =x^{T}\left(t_{k}\right) P\left(t_{k}\right) x\left(t_{k}\right)=\left[\bar{D}_{11} x\left(t_{k}^{-}\right)+\bar{D}_{12} y\left(t_{k}^{-}\right)\right]^{T} P_{2}\left[\bar{D}_{11} x\left(t_{k}^{-}\right)+\bar{D}_{12} y\left(t_{k}^{-}\right)\right] \\
& \leq \zeta^{T}\left(t_{k}^{-}\right) \Psi_{1} \zeta\left(t_{k}^{-}\right)+\mu x^{T}\left(t_{k}^{-}\right) P_{1} x\left(t_{k}^{-}\right)+\gamma_{1} y^{T}\left(t_{k}^{-}\right) Q_{1} y\left(t_{k}^{-}\right) \leq \mu V\left(t_{k}^{-}\right)+\gamma_{1} W\left(t_{k}^{-}\right), \quad k \in \mathbb{N},
\end{aligned}
$$

and

$$
\begin{aligned}
W\left(t_{k}\right) & =y^{T}\left(t_{k}\right) Q\left(t_{k}\right) y\left(t_{k}\right)=\left[\bar{D}_{21} x\left(t_{k}^{-}\right)+\bar{D}_{22} y\left(t_{k}^{-}\right)\right]^{T} Q_{2}\left[\bar{D}_{21} x\left(t_{k}^{-}\right)+\bar{D}_{22} y\left(t_{k}^{-}\right)\right] \\
& \leq \zeta^{T}\left(t_{k}^{-}\right) \Psi_{2} \zeta\left(t_{k}^{-}\right)+\gamma_{2} x^{T}\left(t_{k}^{-}\right) P_{1} x\left(t_{k}^{-}\right)+v y^{T}\left(t_{k}^{-}\right) Q_{1} y\left(t_{k}^{-}\right) \leq \gamma_{2} V\left(t_{k}^{-}\right)+v W\left(t_{k}^{-}\right), \quad k \in \mathbb{N},
\end{aligned}
$$

which implies that the inequality (15) holds. 
Remark 4. By employing the impulse-dependent vector Lyapunov function (7)-(8), the state evolution between two successive impulsive instants is depicted by matrix $C(\varepsilon)$, while the state jumps at the impulse instants are characterized by matrix J. It is worth to highlight that matrix $C(\varepsilon)$ is not Hurwitz stable since $\alpha_{1}>0$ and matrix $J$ is not Schur stable since $v>1$.

We note that the matrices $P(t)$ and $Q(t)$ are both explicitly used in the proof of Lemma 1 and in the conditions (9)-(11). Although Lemma 1 provides some sufficient stability conditions they are non-trivial to check due to the time-varying nature of the matrices $P(t)$ and $Q(t)$ that depend on the sequence $\left(t_{k}\right)_{k \geq 0}$. Thus, in the sequel, we focus on the formulation of a practical method to verify (9)-(11). This will be done by checking a set of LMIs, which do not need a priori knowledge of the sequence $\left(t_{k}\right)_{k \geq 0}$.

Proposition 1. If there exist positive definite matrices $P_{i}(i=1,2)$ and $Q_{i}(i=1,2)$, and some positive constants $\alpha_{i}(i=1,2)$ and $\beta_{j}(j=1,2,3)$, such that the following matrix inequalities hold

$$
\begin{aligned}
& \Phi_{i j}^{1} \triangleq\left[\begin{array}{cc}
\Omega_{i j}^{1} & P_{i} A_{12} \\
* & -\alpha_{2} Q_{i}
\end{array}\right]<0, \\
& \Phi_{i}^{2} \triangleq \operatorname{He}\left\{Q_{i} A_{22}\right\}+\beta_{1} Q_{i}<0, \\
& \Phi_{i j}^{3} \triangleq\left[\begin{array}{cc}
-\beta_{3} P_{i} & \left(L \bar{A}_{11}\right)^{T} Q_{i} \\
* & \Omega_{i j}^{2}
\end{array}\right]<0,
\end{aligned}
$$

where $\Omega_{i j}^{1}=\operatorname{He}\left\{P_{i} \bar{A}_{11}\right\}-\alpha_{1} P_{i}+\frac{P_{1}-P_{2}}{\bar{\tau}_{j}}, \Omega_{i j}^{2}=\operatorname{He}\left\{Q_{i} L A_{12}\right\}-\beta_{2} Q_{i}+\frac{Q_{1}-Q_{2}}{\bar{\tau}_{j}}$. Then the matrix inequalities (9)-(11) hold.

Proof. To show condition (9) we proceed to a convex decomposition of $\Phi^{1}(t)$ as follows

$$
\begin{aligned}
\Phi^{1}(t)= & {\left[\begin{array}{cc}
\operatorname{He}\left\{P(t) \bar{A}_{11}\right\}-\alpha_{1} P(t)+\frac{P_{1}-P_{2}}{\tau_{k+1}} & P(t) A_{12} \\
* & -\alpha_{2} Q(t)
\end{array}\right] } \\
= & (1-\rho(t))\left[\begin{array}{cc}
\operatorname{He}\left\{P_{1} \bar{A}_{11}\right\}-\alpha_{1} P_{1}+\frac{P_{1}-P_{2}}{\tau_{k+1}} & P_{1} A_{12} \\
* & -\alpha_{2} Q_{1}
\end{array}\right] \\
& +\rho(t)\left[\begin{array}{cc}
\operatorname{He}\left\{P_{2} \bar{A}_{11}\right\}-\alpha_{1} P_{2}+\frac{P_{1}-P_{2}}{\tau_{k+1}} & P_{2} A_{12} \\
* & -\alpha_{2} Q_{2}
\end{array}\right] \\
= & (1-\rho(t)) \varrho(t) \Phi_{11}^{1}+(1-\rho(t))(1-\varrho(t)) \Phi_{12}^{1} \\
& +\rho(t) \varrho(t) \Phi_{21}^{1}+\rho(t)(1-\varrho(t)) \Phi_{22}^{1},
\end{aligned}
$$

where $\varrho(t)=\left\{\begin{array}{l}\frac{1 / \tau_{k+1}-1 / \bar{\tau}_{2}}{1 / \bar{\tau}_{1}-1 / \bar{\tau}_{2}} \text { if } \bar{\tau}_{1} \neq \bar{\tau}_{2} \\ 0 \quad \text { otherwise }\end{array}\right.$ and use the fact that $\frac{1}{\tau_{k+1}}=\frac{\varrho(t)}{\bar{\tau}_{1}}+\frac{1-\varrho(t)}{\bar{\tau}_{2}}$.

Consequently, condition (9) holds as far as condition (20) holds true. Similarly, it can be deduced from conditions (21) and (22) that

$$
\Phi^{i}(t)<0, \quad i=2,3 .
$$


Lemma 2 ([12]). Let $V(t, x)=\left[\begin{array}{lll}V_{1}(t, x) & \cdots & V_{n}(t, x)\end{array}\right]^{T}: \mathbb{R}_{+} \times \mathbb{R}^{n} \mapsto \mathbb{R}_{+}^{n}$ and $V_{i}(t, x) \in \mathcal{V}_{0}, i=$ $1,2, \ldots, n$. Assume that

$$
\begin{aligned}
\dot{V}(t, x) & \leq g(t, V(t, x)), t \in\left[t_{k}, t_{k+1}\right), \\
V\left(t, x+I_{k}(x)\right) & \leq \psi_{k}(V(t, x)), t=t_{k}, k \in \mathbb{N},
\end{aligned}
$$

where $g(t, u)=\left[\begin{array}{lll}g_{1}(t, u) & \cdots & g_{n}(t, u)\end{array}\right]^{T}: \mathbb{R}_{+} \times \mathbb{R}^{n} \mapsto \mathbb{R}^{n}$ is quasimonotone nondecreasing in $u$, $I_{k}: \mathbb{R}^{n} \mapsto \mathbb{R}^{n}$ and $\psi_{k}: \mathbb{R}^{n} \mapsto \mathbb{R}_{+}^{n}$ is nondecreasing. Let $r(t)=r\left(t, t_{0}, u_{0}\right)$ be the maximal solution of impulsive differential system

$$
\begin{aligned}
\dot{u} & =g(t, u), t \in\left[t_{k}, t_{k+1}\right), \\
u\left(t_{k}^{+}\right) & =\psi_{k}\left(u\left(t_{k}\right)\right), k \in \mathbb{N}, \quad u\left(t_{0}^{+}\right)=u_{0} \geq 0 .
\end{aligned}
$$

existing on $\left[t_{0}, \infty\right)$. Then $V\left(t_{0}, x_{0}\right) \leq u_{0}$ implies that $V(t, x(t)) \leq r(t), t \geq t_{0}$, where $x(t)=$ $x\left(t, t_{0}, x_{0}\right)$ is any solution of

$$
\begin{aligned}
\dot{x} & =f(t, x), t \in\left[t_{k}, t_{k+1}\right), \\
x\left(t_{k}^{+}\right) & =x\left(t_{k}\right)+I_{k}\left(x\left(t_{k}\right)\right), k \in \mathbb{N}, \quad x\left(t_{0}^{+}\right)=x_{0} \geq 0 .
\end{aligned}
$$

existing on $\left[t_{0}, \infty\right)$.

Lemma 3. Consider the following 2-dimensional vector impulsive comparison system:

$$
\begin{aligned}
\dot{U}(t) & =C(\varepsilon) U(t), t \in\left[t_{k}, t_{k+1}\right), \\
U\left(t_{k}\right) & =J U\left(t_{k}^{-}\right), k \in \mathbb{N},
\end{aligned}
$$

where $C(\varepsilon)=\left[\begin{array}{cc}\alpha_{1} & \alpha_{2} \\ \beta_{3} & \beta_{2}-\beta_{1} / \varepsilon\end{array}\right]$ and $J=\left[\begin{array}{cc}\mu & \gamma_{1} \\ \gamma_{2} & v\end{array}\right]$ with the positive constants $\alpha_{i}, \gamma_{i}, i=1,2, \beta_{j}, j=$ $1,2,3, \varepsilon, \mu \in(0,1)$ and $v>1$. Then, the following facts hold.

(1) $\lim _{\varepsilon \rightarrow 0} \lambda_{\max }(C(\varepsilon))=\alpha_{1}$ and $\frac{d \lambda_{\max }(C(\varepsilon))}{d \varepsilon}>0$;

(2) $e^{C(\varepsilon) t} \leq e^{\lambda_{\max }(C(\varepsilon)) \tau_{2}} \bar{C}(\varepsilon)$, where $\bar{C}(\varepsilon)=\left[\begin{array}{cc}1 & \bar{c}_{12}(\varepsilon) \\ \bar{c}_{21}(\varepsilon) & \bar{c}_{22}(\varepsilon)\end{array}\right], \bar{c}_{12}(\varepsilon)=\frac{\alpha_{2}}{\sqrt{\Delta(\varepsilon)}}, \bar{c}_{21}(\varepsilon)=\frac{\beta_{3}}{\sqrt{\Delta(\varepsilon)}}$, $\bar{c}_{22}(\varepsilon)=\frac{\sqrt{\Delta(\varepsilon)+\kappa_{1}(\varepsilon)}}{2 \sqrt{\Delta(\varepsilon)}}+\frac{\sqrt{\Delta(\varepsilon)}-\kappa_{1}(\varepsilon)}{2 \sqrt{\Delta(\varepsilon)}} e^{-\sqrt{\Delta(\varepsilon) \tau_{1}}}, \kappa_{1}(\varepsilon)=\beta_{2}-\beta_{1} / \varepsilon-\alpha_{1}$, and $\Delta(\varepsilon)=\kappa_{1}^{2}(\varepsilon)+4 \alpha_{2} \beta_{3} ;$

(3) If $\beta_{2}>\alpha_{1}$ and there exists $\varepsilon^{*}>0$ such that the following condition holds,

$$
\theta\left(\varepsilon^{*}\right) \triangleq e^{\lambda_{\max }\left(C\left(\varepsilon^{*}\right)\right) \tau_{2}} \varrho\left(J \bar{C}\left(\varepsilon^{*}\right)\right)<1,
$$

where $\varrho(\cdot)$ denotes the spectral radius of matrix, then system (29)-(30) is GUES for any $\varepsilon \in\left(0, \varepsilon^{*}\right)$.

Proof. Straightforward computation gives that

$$
\left.\begin{array}{l}
\lambda_{\max }(C(\varepsilon))=\frac{1}{2}\left[\kappa_{2}(\varepsilon)+\sqrt{\Delta(\varepsilon)}\right] \\
\lambda_{\min }(C(\varepsilon))=\frac{1}{2}\left[\kappa_{2}(\varepsilon)-\sqrt{\Delta(\varepsilon)}\right]
\end{array}\right\} \Rightarrow \lambda_{\max }(C(\varepsilon))-\lambda_{\min }(C(\varepsilon))=\sqrt{\Delta(\varepsilon)},
$$

where $\kappa_{2}(\varepsilon)=\beta_{2}-\beta_{1} / \varepsilon+\alpha_{1}$. Moreover, the eigenvectors associated with $\lambda_{\max }(C(\varepsilon))$ and $\lambda_{\min }(C(\varepsilon))$ are $\left[\frac{2 \alpha_{2}}{\overline{\kappa_{1}(\varepsilon)+\sqrt{\Delta(\varepsilon)}}} \quad 1\right]^{T}$ and $\left[\frac{2 \alpha_{2}}{\overline{\kappa_{1}(\varepsilon)-\sqrt{\Delta(\varepsilon)}}} 8\right]^{T}$, respectively. 
(1) It is easy to deduce that

$$
\begin{aligned}
\lim _{\varepsilon \rightarrow 0} \lambda_{\max }(C(\varepsilon)) & =\frac{1}{2} \lim _{\varepsilon \rightarrow 0} \frac{\left(\sqrt{\Delta(\varepsilon)}+\kappa_{2}(\varepsilon)\right)\left(\sqrt{\Delta(\varepsilon)}-\kappa_{2}(\varepsilon)\right)}{\sqrt{\Delta(\varepsilon)}-\kappa_{2}(\varepsilon)}=\frac{1}{2} \lim _{\varepsilon \rightarrow 0} \frac{\Delta(\varepsilon)-\kappa_{2}^{2}(\varepsilon)}{\sqrt{\Delta(\varepsilon)}-\kappa_{2}(\varepsilon)} \\
& =\frac{1}{2} \lim _{\varepsilon \rightarrow 0} \frac{\kappa_{1}^{2}(\varepsilon)+4 \alpha_{2} \beta_{3}-\kappa_{2}^{2}(\varepsilon)}{\sqrt{\Delta(\varepsilon)}-\kappa_{2}(\varepsilon)}=\frac{1}{2} \lim _{\varepsilon \rightarrow 0} \frac{4 \alpha_{2} \beta_{3}-4 \alpha_{1}\left(\beta_{2}-\beta_{1} / \varepsilon\right)}{\sqrt{\Delta(\varepsilon)}-\kappa_{2}(\varepsilon)} \\
& =\frac{1}{2} \frac{4 \alpha_{1} \beta_{1} / \varepsilon}{\beta_{1} / \varepsilon+\beta_{1} / \varepsilon}=\alpha_{1},
\end{aligned}
$$

and the derivative of $\lambda_{\max }(C(\varepsilon))$ with respect to $\varepsilon$ is

$$
\begin{aligned}
\frac{d \lambda_{\max }(C(\varepsilon))}{d \varepsilon} & =\frac{1}{2}\left[\frac{d \kappa_{2}(\varepsilon)}{d \varepsilon}+\frac{d \sqrt{\Delta(\varepsilon)}}{d \varepsilon}\right]=\frac{1}{2}\left[\frac{\beta_{1}}{\varepsilon^{2}}+\frac{1}{2} \frac{1}{\sqrt{\Delta(\varepsilon)}} \frac{d \Delta(\varepsilon)}{d \varepsilon}\right] \\
& =\frac{1}{2}\left[\frac{\beta_{1}}{\varepsilon^{2}}+\frac{1}{2} \frac{1}{\sqrt{\Delta(\varepsilon)}} 2 \kappa_{1}(\varepsilon) \frac{\beta_{1}}{\varepsilon^{2}}\right]=\frac{\beta_{1}}{2 \sqrt{\Delta(\varepsilon)} \varepsilon^{2}}\left[\sqrt{\Delta(\varepsilon)}+\kappa_{1}(\varepsilon)\right]>0, \quad \forall \varepsilon>0 .
\end{aligned}
$$

(2) It is also straightforward that

$$
e^{C(\varepsilon) t}=M\left[\begin{array}{cc}
e^{\lambda_{\max }(C(\varepsilon)) t} & 0 \\
0 & e^{\lambda_{\min }(C(\varepsilon)) t}
\end{array}\right] M^{-1}=e^{\lambda_{\max }(C(\varepsilon)) t} M\left[\begin{array}{cc}
1 & 0 \\
0 & e^{\sqrt{\Delta(\varepsilon) t}}
\end{array}\right] M^{-1},
$$

where $M=\left[\begin{array}{cc}\frac{2 \alpha_{2}}{\kappa_{1}(\varepsilon)+\sqrt{\Delta(\varepsilon)}} & \frac{2 \alpha_{2}}{\kappa_{1}(\varepsilon)-\sqrt{\Delta(\varepsilon)}} \\ 1 & 1\end{array}\right], M^{-1}=\left[\begin{array}{cc}\frac{\beta_{3}}{\sqrt{\Delta(\varepsilon)}} & \sqrt{\Delta(\varepsilon)}+\frac{\kappa_{1}(\varepsilon)}{2 \sqrt{\Delta(\varepsilon)}} \\ -\frac{\beta_{3}}{\sqrt{\Delta(\varepsilon)}} & \frac{\sqrt{\Delta(\varepsilon)}-\kappa_{1}(\varepsilon)}{2 \sqrt{\Delta(\varepsilon)}}\end{array}\right]$.

Therefore, it can be deduced that

$$
e^{C(\varepsilon) t}=e^{\lambda_{\max }(C(\varepsilon)) t}\left[\begin{array}{ll}
c_{11}(\varepsilon, t) & c_{12}(\varepsilon, t) \\
c_{21}(\varepsilon, t) & c_{22}(\varepsilon, t)
\end{array}\right]
$$

with

$$
\begin{aligned}
& c_{11}(\varepsilon, t)=\frac{2 \alpha_{2} \beta_{3}}{\sqrt{\Delta(\varepsilon)}}\left[\frac{1}{\sqrt{\Delta(\varepsilon)}+\kappa_{1}(\varepsilon)}+\frac{e^{-\sqrt{\Delta(\varepsilon)} t}}{\sqrt{\Delta(\varepsilon)}-\kappa_{1}(\varepsilon)}\right], \\
& c_{12}(\varepsilon, t)=\frac{\alpha_{2}}{\sqrt{\Delta(\varepsilon)}}\left[1-e^{-\sqrt{\Delta(\varepsilon) t}}\right], c_{21}(\varepsilon, t)=\frac{\beta_{3}}{\sqrt{\Delta(\varepsilon)}}\left[1-e^{-\sqrt{\Delta(\varepsilon) t}}\right], \\
& c_{22}(\varepsilon, t)=\frac{\sqrt{\Delta(\varepsilon)}+\kappa_{1}(\varepsilon)}{2 \sqrt{\Delta(\varepsilon)}}+\frac{\sqrt{\Delta(\varepsilon)}-\kappa_{1}(\varepsilon)}{2 \sqrt{\Delta(\varepsilon)}} e^{-\sqrt{\Delta(\varepsilon) t}} .
\end{aligned}
$$

We notice that

$$
\begin{aligned}
& c_{11}(\varepsilon, t) \leq \frac{2 \alpha_{2} \beta_{3}}{\sqrt{\Delta(\varepsilon)}}\left[\frac{1}{\sqrt{\Delta(\varepsilon)}+\kappa_{1}(\varepsilon)}+\frac{1}{\sqrt{\Delta(\varepsilon)}-\kappa_{1}(\varepsilon)}\right]=\frac{2 \alpha_{2} \beta_{3}}{\sqrt{\Delta(\varepsilon)}} \frac{2 \sqrt{\Delta(\varepsilon)}}{\Delta(\varepsilon)-\kappa_{1}^{2}(\varepsilon)}=\frac{2 \alpha_{2} \beta_{3}}{\sqrt{\Delta(\varepsilon)}} \frac{2 \sqrt{\Delta(\varepsilon)}}{4 \alpha_{2} \beta_{3}}=1, \\
& c_{12}(\varepsilon, t) \leq \frac{\alpha_{2}}{\sqrt{\Delta(\varepsilon)}}=\bar{c}_{12}(\varepsilon), \\
& c_{21}(\varepsilon, t) \leq \frac{\beta_{3}}{\sqrt{\Delta(\varepsilon)}}=\bar{c}_{21}(\varepsilon), \\
& c_{22}(\varepsilon, t) \leq \frac{\sqrt{\Delta(\varepsilon)}+\kappa_{1}(\varepsilon)}{2 \sqrt{\Delta(\varepsilon)}}+\frac{\sqrt{\Delta(\varepsilon)}-\kappa_{1}(\varepsilon)}{2 \sqrt{\Delta(\varepsilon)}} e^{-\sqrt{\Delta(\varepsilon)} \tau_{1}}=\bar{c}_{22}(\varepsilon) .
\end{aligned}
$$


(3) Since $\beta_{2}>\alpha_{1}$, let $\varepsilon_{1}=\frac{\beta_{1}}{\beta_{2}-\alpha_{1}}$, then $\varepsilon_{1}>0$. Moreover, it is easy to derive that

$$
\kappa_{1}(\varepsilon)=\beta_{2}-\alpha_{1}-\beta_{1} / \varepsilon<0, \forall \varepsilon<\varepsilon_{1},
$$

and

$$
\frac{d \Delta^{-1 / 2}(\varepsilon)}{d \varepsilon}=-\frac{1}{2} \Delta^{-3 / 2}(\varepsilon) \frac{d \Delta(\varepsilon)}{d \varepsilon}=-\frac{1}{2 \Delta^{3 / 2}(\varepsilon)} \times 2 \kappa_{1}(\varepsilon) \frac{\beta_{1}}{\varepsilon^{2}}=-\frac{\beta_{1} \kappa_{1}(\varepsilon)}{\Delta^{3 / 2}(\varepsilon) \varepsilon^{2}}>0, \forall \varepsilon<\varepsilon_{1} .
$$

Thus, for any $\forall \varepsilon<\varepsilon_{1}$, the derivatives of $\bar{c}_{12}(\varepsilon)$ and $\bar{c}_{21}(\varepsilon)$ with respect to $\varepsilon$ are

$$
\begin{aligned}
\frac{d \bar{c}_{12}(\varepsilon)}{d \varepsilon}= & -\frac{\alpha_{2} \beta_{1}}{\Delta^{3 / 2}(\varepsilon) \varepsilon^{2}} \kappa_{1}(\varepsilon)>0, \\
\frac{d \bar{c}_{21}(\varepsilon)}{d \varepsilon}= & -\frac{\beta_{3} \beta_{1}}{\Delta(\varepsilon) \sqrt{\Delta(\varepsilon)} \varepsilon^{2}} \kappa_{1}(\varepsilon)>0, \\
\frac{d \bar{c}_{22}(\varepsilon)}{d \varepsilon}= & \frac{1}{2} \frac{d\left\{\frac{\kappa_{1}(\varepsilon)}{\sqrt{\Delta(\varepsilon)}}\right\}}{d \varepsilon}-\frac{1}{2} \frac{d\left\{\frac{\kappa_{1}(\varepsilon)}{\sqrt{\Delta(\varepsilon)}}\right\}}{d \varepsilon} e^{-\sqrt{\Delta(\varepsilon)} \tau_{1}}+\frac{1}{2}\left\{1-\frac{\kappa_{1}(\varepsilon)}{\sqrt{\Delta(\varepsilon)}}\right\} \frac{d\left\{e^{-\sqrt{\Delta(\varepsilon)} \tau_{1}}\right\}}{d \varepsilon} \\
= & \frac{1}{2} \frac{\frac{d \kappa_{1}(\varepsilon)}{d \varepsilon} \sqrt{\Delta(\varepsilon)}-\kappa_{1}(\varepsilon) \frac{d \sqrt{\Delta(\varepsilon)}}{d \varepsilon}}{\Delta(\varepsilon)}-\frac{1}{2} \frac{\frac{d \kappa_{1}(\varepsilon)}{d \varepsilon} \sqrt{\Delta(\varepsilon)}-\kappa_{1}(\varepsilon) \frac{d \sqrt{\Delta(\varepsilon)}}{d \varepsilon}}{\Delta(\varepsilon)} e^{-\sqrt{\Delta(\varepsilon)} \tau_{1}} \\
& -\frac{\tau_{1}}{2}\left\{1-\frac{\kappa_{1}(\varepsilon)}{\sqrt{\Delta(\varepsilon)}}\right\} e^{-\sqrt{\Delta(\varepsilon)} \tau_{1}} \frac{d \sqrt{\Delta(\varepsilon)}}{d \varepsilon} \\
= & \frac{1}{2} \frac{\frac{\beta_{1}}{\varepsilon^{2}} \sqrt{\Delta(\varepsilon)}-\frac{\kappa_{1}^{2}(\varepsilon)}{\sqrt{\Delta(\varepsilon)}} \frac{\beta_{1}}{\varepsilon^{2}}}{\Delta(\varepsilon)}\left[1-e^{-\sqrt{\Delta(\varepsilon)} \tau_{1}}\right]-\frac{\tau_{1}}{2} \frac{\sqrt{\Delta(\varepsilon)}-\kappa_{1}(\varepsilon)}{\Delta(\varepsilon)} e^{-\sqrt{\Delta(\varepsilon)} \tau_{1}} \frac{\kappa_{1}(\varepsilon) \beta_{1}}{\varepsilon^{2}} \\
= & \frac{2 \alpha_{2} \beta_{1} \beta_{3}}{\Delta^{3 / 2} \varepsilon^{2}}\left[1-e^{-\sqrt{\Delta(\varepsilon)} \tau_{1}}\right]+\frac{\beta_{1} \tau_{1}}{2 \Delta(\varepsilon) \varepsilon^{2}}\left[\kappa_{1}(\varepsilon)-\sqrt{\Delta(\varepsilon)}\right] \kappa_{1}(\varepsilon) e^{-\sqrt{\Delta(\varepsilon)} \tau_{1}}>0 .
\end{aligned}
$$

Consequently, there exists $0<\varepsilon^{*} \leq \varepsilon_{1}$ such that for any $t \in\left[t_{0}, t_{1}\right)$ and any $\varepsilon<\varepsilon^{*}$

$$
U(t)=e^{C(\varepsilon) t} U\left(t_{0}\right) \leq e^{\lambda_{\max }\left(C\left(\varepsilon^{*}\right)\right) \tau_{2}} \bar{C}\left(\varepsilon^{*}\right) U\left(t_{0}\right)
$$

and

$$
U\left(t_{1}\right)=J U\left(t_{1}^{-}\right) \leq e^{\lambda_{\max }\left(C\left(\varepsilon^{*}\right)\right) \tau_{2}} J \bar{C}\left(\varepsilon^{*}\right) U\left(t_{0}\right) .
$$

Moreover, based on the condition (31), it can be achieved recursively that

$$
\begin{aligned}
U(t) & \leq e^{\lambda_{\max }\left(C\left(\varepsilon^{*}\right)\right) \tau_{2}} \bar{C}\left(\varepsilon^{*}\right)\left(e^{\lambda_{\max }\left(C\left(\varepsilon^{*}\right)\right) \tau_{2}} J \bar{C}\left(\varepsilon^{*}\right)\right)^{k} U\left(t_{0}\right) \leq e^{\lambda_{\max }\left(C\left(\varepsilon^{*}\right)\right) \tau_{2}} \bar{C}\left(\varepsilon^{*}\right) \theta^{k}\left(\varepsilon^{*}\right) U\left(t_{0}\right) \\
& \leq e^{\lambda_{\max }\left(C\left(\varepsilon^{*}\right)\right) \tau_{2}} \bar{C}\left(\varepsilon^{*}\right) e^{k \ln \left(\theta\left(\varepsilon^{*}\right)\right)} U\left(t_{0}\right) \leq \frac{e^{\lambda_{\max }\left(C\left(\varepsilon^{*}\right)\right) \tau_{2}} \bar{C}\left(\varepsilon^{*}\right)}{\theta\left(\varepsilon^{*}\right)} e^{\frac{\left.\ln \left(\theta \varepsilon^{*}\right)\right)}{\tau_{2}}\left(t-t_{0}\right)} U\left(t_{0}\right), \quad \forall t \in\left[t_{k}, t_{k+1}\right),
\end{aligned}
$$

which means that system (29)-(30) is GUES for any $\varepsilon \in\left(0, \varepsilon^{*}\right)$.

Remark 5. Assertions (1)-(2) of Lemma 3 show that the flow dynamic is unstable and the divergence of system states is related to the parameters $\alpha_{1}$, the upper and the lower bound of impulsive time interval and matrix $\bar{C}(\varepsilon)$. Assertion (3) of Lemma 3 shows that the stability of the 
2-dimensional vector impulsive comparison system (29)-(30) can be imposed by mixed impulses satisfying appropriate properties. At this point it is interesting to emphasize that Lemma 3 can be used to design the impulse dynamics that stabilizes the original system but this is out of our scope in this paper. Finally, it is noteworthy that the upper bound of the singular perturbation parameter has an important effect on the stability.

Based on Lemmas 1-3, we are in the position to present the GUES of system (1)-(2).

Theorem 1. Consider the continuous-time impulsive system (1)-(2) or (4)-(5) and suppose that Assumption 1 holds. Let $\bar{\tau}_{1}$ and $\bar{\tau}_{2}$ be given positive scalars satisfying $\bar{\tau}_{1} \leq \bar{\tau}_{2}$. If, for all $i \in\{1,2\} j \in\{1,2,3\}$ and $k \in\{1,2\}$, there exist positive definite matrices $P_{i}$ and $Q_{i}$, some positive constants $\alpha_{i}, \beta_{j}, \gamma_{k}, \mu \in(0,1)$ and $v>1$ with $\beta_{2}>\alpha_{1}$, and a positive constant $\varepsilon^{*}$ such that the inequalities (20)-(22), (12)-(13) and (31) hold, then system (1)-(2) or equivqlently (4)-(5) is GUES for any $\varepsilon \in\left(0, \varepsilon^{*}\right)$.

Proof. Inequalities (20)-(22) and Proposition 1 yield that inequalities (9)-(11) of Lemma 1 hold. Consequently, Lemma 1 shows that the GUES problem of system (1)-(2) or (4)-(5) can be reduced to stability analysis of the two-dimensional vector system (14)-(15). Moreover, stability of the system (14)-(15) can be deduced from the stability of the 2-dimensional vector impulsive comparison system (29)-(30) by employing Lemma 2.

Since $\beta_{2}>\alpha_{1}$ and there exists $\varepsilon^{*}>0$ such that the condition (31) holds, based on Lemma 3 , it can be concluded that system (29)-(30) is GUES for any $\varepsilon \in\left(0, \varepsilon^{*}\right)$, which also implies that system (1)-(2) or (4)-(5) is GUES for any $\varepsilon \in\left(0, \varepsilon^{*}\right)$.

Remark 6. The most important feature of the proposed method is its ability to deal with a more challenging case of unstable flow and unstable jump dynamics. On top of that, by using impulse-dependent vector Lyapunov function and vector comparison principle, Theorem 1 gives sufficient stability conditions for system (1)-(2) or (4)-(5), which are numerically tractable if the parameters of matrices $C(\varepsilon)$ and $J$ are a priori given.

How to obtain the upper bound of the singular perturbation parameter is an interesting but challenging problem. Here, we provide a procedure to clearly describe how to obtain numerically the upper bound $\varepsilon^{*}$ :

(1) For given SPISs (1)-(2) and parameter $\bar{\tau}_{i}(i=1,2)$, determine the parameters $\alpha_{i}(i=$ $1,2), \beta_{j}(j=1,2,3), \gamma_{k}(k=1,2), \mu$ and $v$ by solving the inequalities (20)-(22) and (12)(13);

(2) By employing a simple one-dimensional search over $\varepsilon^{*}>0$, we can obtain the upper bound $\varepsilon^{*}$ satisfying the inequality (31).

\section{Simulation}

In the section, the effectiveness of the obtained results is illustrated.

Example 1. Let us consider the system (1)-(2) with the state matrices described by the following numerical values:

$$
\begin{aligned}
& A_{11}=1, A_{12}=-1, A_{21}=2, A_{22}=-3, \\
& D_{11}=0.8, D_{12}=-2, D_{21}=0, D_{22}=1.5 .
\end{aligned}
$$


It is easy to see that $\bar{A}_{11}=A_{11}-A_{12} A_{22}^{-1} A_{21}=1 / 3>0$, that is, the flow dynamic of the system in this example is unstable. We also note that the jump dynamics is described by the mixed impulses with the impulsive magnitudes 0.8 and 1.5 for the slow and the fast dynamics, respectively. Thus, the exiting results on the stability of SPISs cannot be applied here and our methodology has to be employed.

Let us first illustrate the effect of the time interval between consecutive impulse instants on the stability of SPSs with the mixed impulses. It can be seen in Figs. 1 and 2 that the stability of SPSs with mixed impulses is not reached if the impulses occur too frequently or too rarely. We recall here that our objective is not the design of the impulse instants sequence but stability is not guaranteed as far as the interval between impulses does not verify the upper and lower bound constraints.

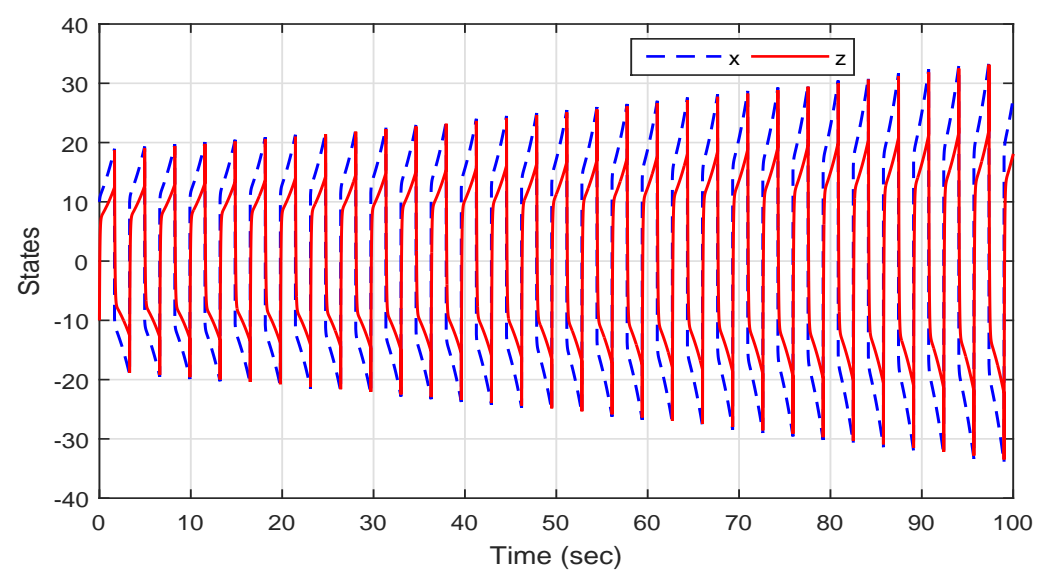

Figure 1: State response of system in Example 1 with $\tau_{1}=\tau_{2}=1.65$.

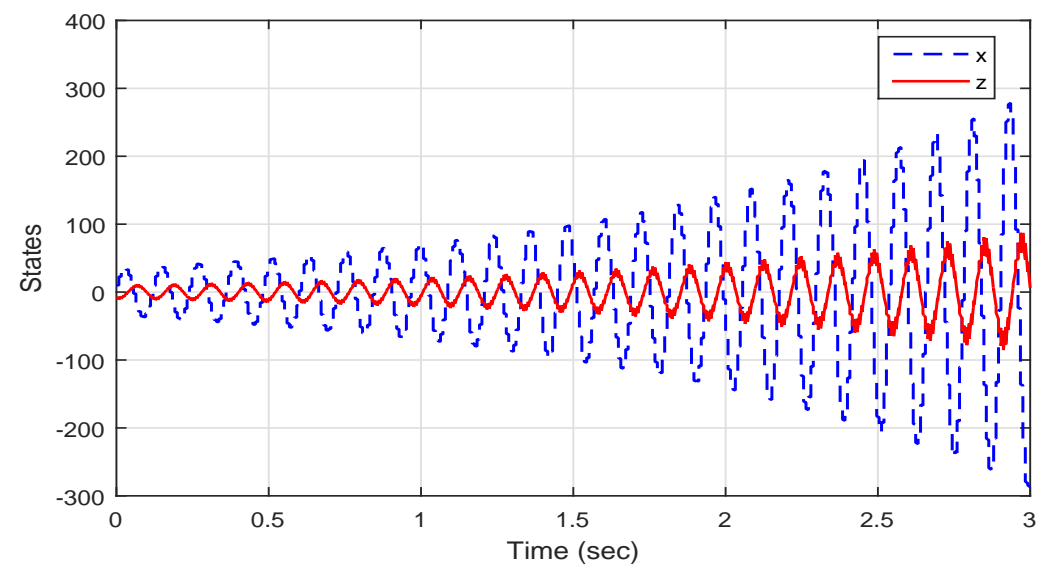

Figure 2: State response of system in Example 1 with $\tau_{1}=\tau_{2}=0.008$. 
Let us illustrate now Theorem 1 when the impulse instants verify our assumptions. To do that we fix $\bar{\tau}_{1}=\bar{\tau}_{2}=1$, i.e., the impulsive time sequence belongs to $\mathcal{N}(1)$. We also fix $\mu=0.23, v=$ $33.066, \beta_{1}=5.999, \beta_{2}=15.982, \beta_{3}=0.175, \gamma_{1}=4.991, \gamma_{2}=2.104, \alpha_{1}=1.405$ and $\alpha_{2}=1.52$, it can be found that all conditions of Theorem 1 hold with

$$
P_{1}=104.0496, P_{2}=81.0744, Q_{1}=1786.8, Q_{2}=117.2405,
$$

and $\varepsilon^{*}=0.1342$. Thus, according to Theorem 1 , the system is GUES for any $\varepsilon \in(0,0.1342)$.

In the simulation, the initial condition is taken as $\left[\begin{array}{ll}10 & -10\end{array}\right]$ and $\varepsilon=0.1341$, the state evolution is given in Figs. 3-4, which shows that the trajectories of the system in Example 1 converge to zero. Moreover, Fig. 4 shows that the fast state will converge to the slow manifold very quickly, and slide on the slow manifold for a while until an impulse occurs. Due to the impulse, the fast state deviates from the slow manifold abruptly. Then, the fast state quickly converges again to the slow manifold, and this process continues until the system trajectory reaches zero.

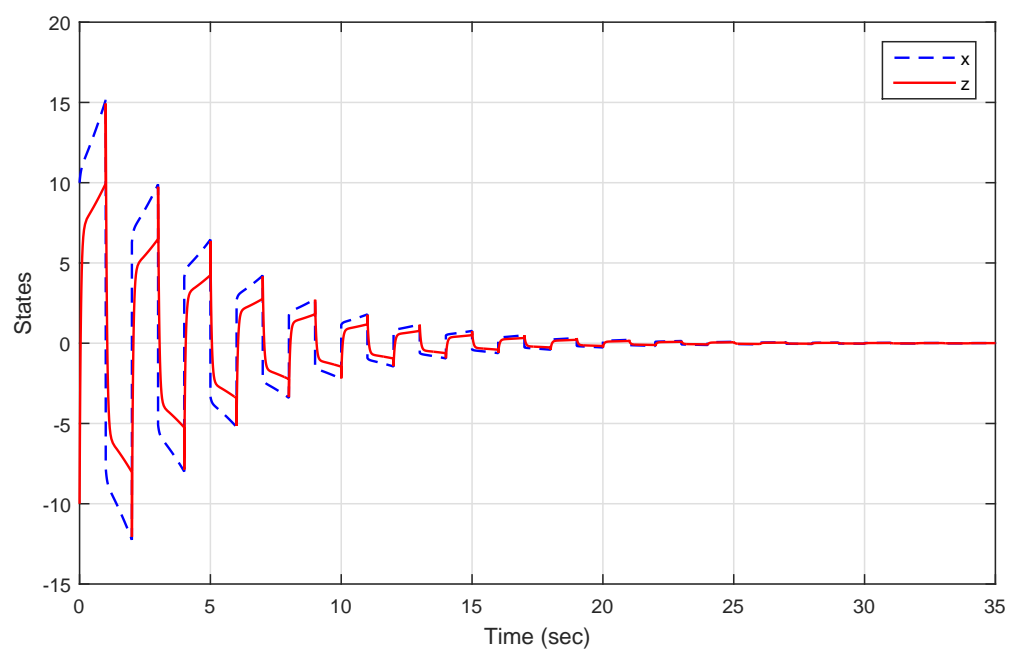

Figure 3: State response of system in Example 1 with $\varepsilon=0.1341$.

Moreover, Theorem 1 shows that the impulsive time sequence can be aperiodic. Select $\bar{\tau}_{1}=$ $1, \bar{\tau}_{2}=1.04$, i.e., the impulsive time sequence belongs to $\mathcal{N}(1,1.04)$, and the others parameters are defined in above analysis, it can be found that all conditions of Theorem 1 hold with $\varepsilon^{*}=$ 0.0537. Thus, according to Theorem 1, the system in this example can achieve GUES for any $\varepsilon \in(0,0.0537)$.

Example 2. This example is to illustrate the proposed stability result for a higher-dimensional SPSs with mixed impulses. Let us reconsider system (1)-(2) when the state matrices take the following numerical values:

$$
\begin{aligned}
A_{11} & =\left[\begin{array}{cc}
0.3 & 0.1 \\
0.05 & -0.65
\end{array}\right], A_{12}=\left[\begin{array}{cc}
0 & 0 \\
0.345 & 0
\end{array}\right], A_{21}=\left[\begin{array}{cc}
0.1 & -0.524 \\
0.7 & 0.23
\end{array}\right], A_{22}=\left[\begin{array}{cc}
-2.465 & 0.262 \\
0.1 & -2.1
\end{array}\right], \\
D_{11} & =\left[\begin{array}{cc}
0.25 & 0 \\
0 & 0.25
\end{array}\right], D_{12}=\left[\begin{array}{cc}
1 & -1 \\
-1 & 1
\end{array}\right], D_{21}=\left[\begin{array}{ll}
0 & 0 \\
0 & 0
\end{array}\right], D_{22}=\left[\begin{array}{cc}
1.5 & 0 \\
0 & 1.5
\end{array}\right] .
\end{aligned}
$$




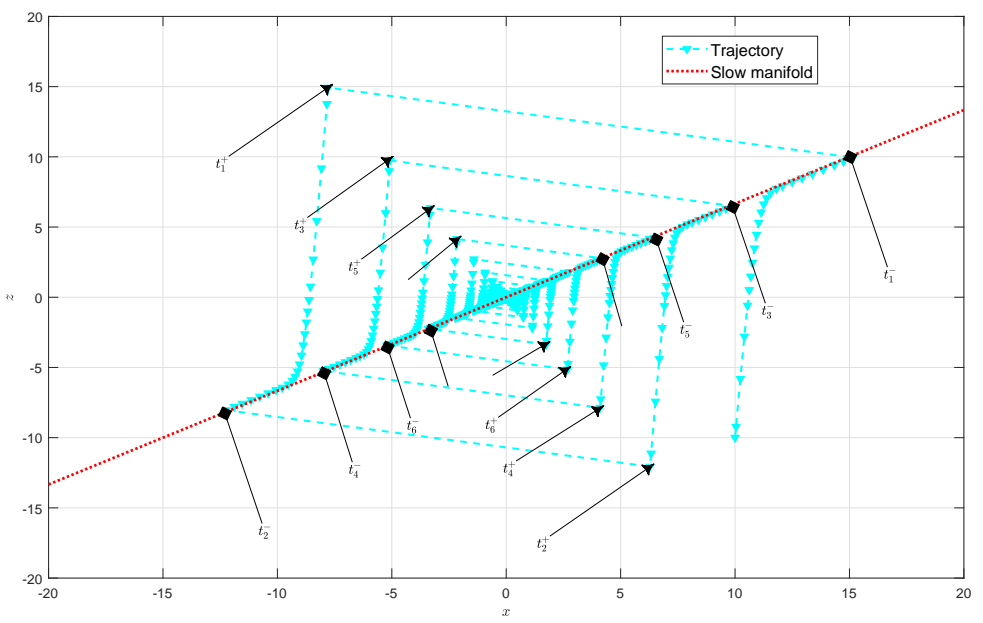

Figure 4: State response of system in Example 1 with $\varepsilon=0.1341$, where $t_{k}^{-}$and $t_{k}^{+}$are denoted by the black diamond and the black triangle, respectively.

It can be deduced that $\bar{A}_{11}=\left[\begin{array}{cc}0.3000 & 0.1000 \\ 0.0764 & -0.7197\end{array}\right]$, and the eigenvalues of $\bar{A}_{11}$ are 0.3074 and -0.7271 , which means that the flow dynamics of the system under consideration is unstable. Let us consider in the following that mixed impulses are affecting the flow dynamics. Specifically, the slow and the fast dynamics are affected by mixed impulses with the impulsive magnitudes 0.25 and 1.5, respectively. To fit the setup of Theorem 1, we fix the impulsive time interval $\bar{\tau}_{1}=\bar{\tau}_{2}=$ 1 , and let $\mu=0.10, v=7.68, \beta_{1}=4.00, \beta_{2}=4.91, \beta_{3}=0.10, \gamma_{1}=4.00, \gamma_{2}=2.00, \alpha_{1}=0.02$ and $\alpha_{2}=0.23$. Computing the upper bound on the singular perturbation parameter we find $\varepsilon^{*}=0.4937$ meaning that the system in this example is GUES for any $\varepsilon \in(0,0.4937)$.

In the following simulation we fix the singular perturbation parameter $\varepsilon=0.4936$ and the initial condition as $\left[\begin{array}{llll}0 & -10 & 5 & -5\end{array}\right]$. The state evolution is given in Figs. 5-6, which illustrate that the trajectory of the system in Example 2 converges to zero. 


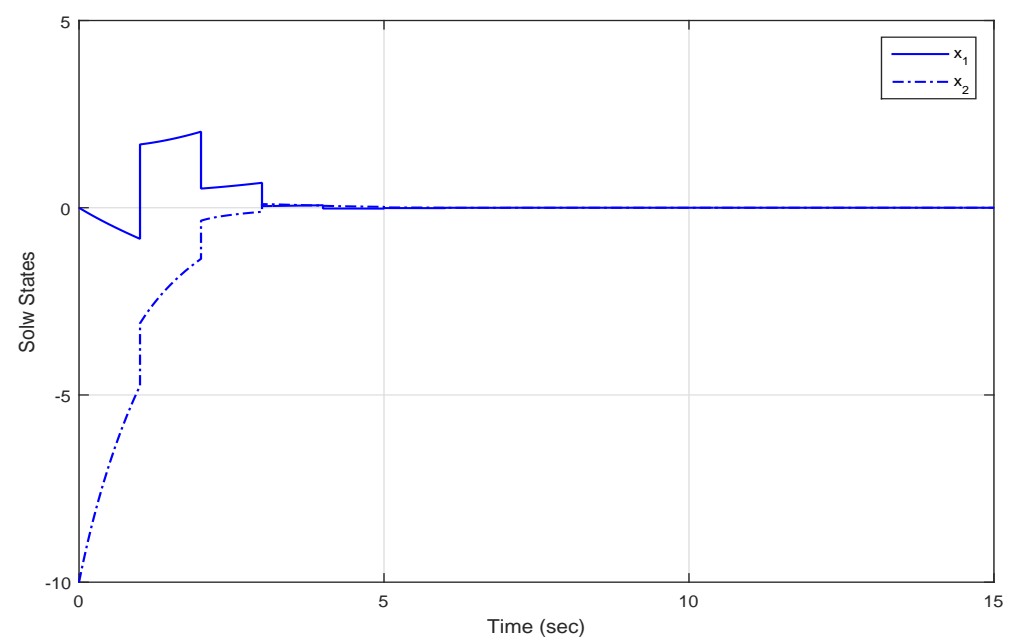

Figure 5: Slow states response of system in Example 2 with $\varepsilon=0.4936$.

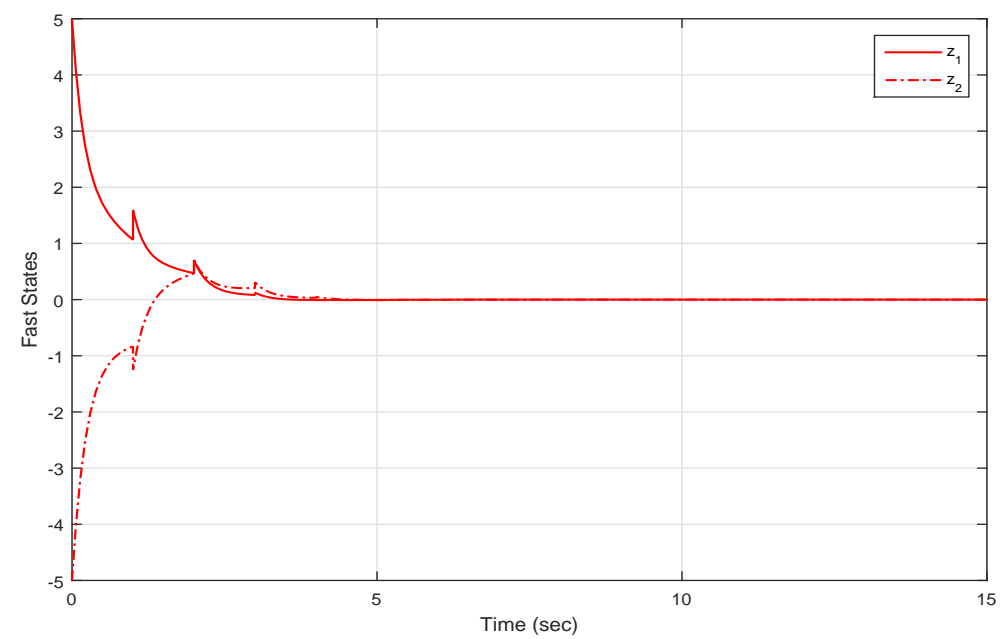

Figure 6: Fast states response of system in Example 2 with $\varepsilon=0.4936$.

\section{Conclusion}

This paper dealt with the situations where the flow dynamic is unstable and exhibits a twotime-scale feature, while the jump dynamic contains both the destabilizing and stabilizing impulses simultaneously. To explore the positive effect of the stabilizing impulse and describe the relationship between two consecutive impulses, a new kind of impulse-dependent vector Lyapunov function has been constructed. Based on vector comparison principle, some sufficient 
conditions have been derived to ensure the GUES of SPSs with mixed impulses. Moreover, the upper bound of singular perturbation parameter has been obtained by using one-dimensional search algorithm. Finally, two examples have been presented to illustrate the effectiveness of the proposed results. Future research will focus on the stability of SPSs with time delay and impulsive effects. Additional research will also target the case of stochastic impulses.

\section{Acknowledgements}

The work of W. Yang was partially supported by the National Natural Science Foundation of China under grant 61903147; The work of Y.-W. Wang was partially supported by the National Natural Science Foundation of China under grant 61773172; The work of I.-C. Morărescu and J. Daafouz was partially supported by ANR via grant HANDY, number ANR-18-CE40-0010; The work of I.-C. Morărescu was also supported by ANR via grant NICETWEET, number ANR-20CE48-0009.

\section{References}

[1] Y. Wang, Y. Gao, H. R. Karimi, H. Shen, Z. Fang, Sliding mode control of fuzzy singularly perturbed systems with application to electric circuit, IEEE Transactions on Systems, Man, and Cybernetics: Systems 48 (10) (2018) 1667-1675.

[2] I. Malloci, J. Daafouz, C. Iung, R. Bonidal, P. Szczepanski, Switched system modeling and robust steering control of the tail end phase in a hot strip mill, Nonlinear Analysis: Hybrid Systems 3 (3) (2009) 239-250.

[3] J. Kim, Two-time scale control of flexible joint robots with an improved slow model, IEEE Transactions on Industrial Electronics 65 (4) (2018) 3317-3325.

[4] W. Yang, Y.-W. Wang, Y. Shen, L. Pan, Cluster synchronization of coupled delayed competitive neural networks with two time scales, Nonlinear Dynamics 90 (4) (2017) 2767-2782.

[5] A. Saberi, H. Khalil, Quadratic-type Lyapunov functions for singularly perturbed systems, IEEE Transactions on Automatic Control 29 (6) (1984) 542-550.

[6] M. Corless, L. Glielmo, On the exponential stability of singularly perturbed systems, SIAM Journal on Control and Optimization 30 (6) (1992) 1338-1360.

[7] E. Fridman, Effects of small delays on stability of singularly perturbed systems, Automatica 38 (5) (2002) $897-902$.

[8] H. Liu, F. Sun, Z. Sun, Stability analysis and synthesis of fuzzy singularly perturbed systems, IEEE Transactions on Fuzzy Systems 13 (2) (2005) 273-284.

[9] A. R. Teel, L. Moreau, D. Nesic, A unified framework for input-to-state stability in systems with two time scales, IEEE Transactions on Automatic Control 48 (9) (2003) 1526-1544.

[10] P. Kokotovic, H. K. Khalil, J. O'reilly, Singular Perturbation Methods in Control: Analysis and Design, SIAM, Philadelphia, PA, 1999.

[11] Y.-W. Wang, W. Yang, X.-K. Liu, W.-H. Chen, Dissipativity of singularly perturbed Lur'e systems, IEEE Transactions on Circuits and Systems II: Express Briefs.

[12] V. Lakshmikantham, D. D. Bainov, P. S. Simeonov, Theory of Impulsive Differential Equations, Singapore: World Scientific, 1989.

[13] Y.-W. Wang, W. Yang, Survey on hybrid singularly perturbed systems, Control and Decision 33 (5) (2018(in Chinese)) 950-959.

[14] W. Yang, Y.-W. Wang, Z.-H. Guan, C. Wen, Controllability of impulsive singularly perturbed systems and its application to a class of multiplex networks, Nonlinear Analysis: Hybrid Systems 31 (2019) 123-134.

[15] P. Simeonov, D. Bainov, Stability of the solutions of singularly perturbed systems with impulse effect, Journal of Mathematical Analysis and Applications 136 (2) (1988) 575-588.

[16] P. Simeonov, D. Bainov, Exponential stability of the solutions of singularly perturbed systems with impulse effect, Journal of Mathematical Analysis and Applications 151 (2) (1990) 462-487.

[17] M. S. Alwan, X. Liu, Stability of singularly perturbed switched systems with time delay and impulsive effects, Nonlinear Analysis: Theory, Methods \& Applications 71 (9) (2009) 4297-4308.

[18] W.-H. Chen, F. Chen, X. Lu, Exponential stability of a class of singularly perturbed stochastic time-delay systems with impulse effect, Nonlinear Analysis: Real World Applications 11 (5) (2010) 3463-3478. 
[19] J. B. Rejeb, I.-C. Morărescu, A. Girard, J. Daafouz, Stability analysis of a general class of singularly perturbed linear hybrid systems, Automatica 90 (2018) 98-108.

[20] J. Lu, D. W. Ho, J. Cao, A unified synchronization criterion for impulsive dynamical networks, Automatica 46 (7) (2010) 1215-1221.

[21] X. Li, D. W. Ho, J. Cao, Finite-time stability and settling-time estimation of nonlinear impulsive systems, Automatica 99 (2019) 361-368.

[22] T. Yang, Impulsive Control Theory, Berlin, Germany: Springer-Verlag, 2001.

[23] Y.-W. Wang, W. Yang, J.-W. Xiao, Z.-G. Zeng, Impulsive multisynchronization of coupled multistable neural networks with time-varying delay, IEEE Transactions on Neural Networks and Learning Systems 28 (7) (2017) 1560-1571.

[24] D. Yang, X. Li, J. Qiu, Output tracking control of delayed switched systems via state-dependent switching and dynamic output feedback, Nonlinear Analysis: Hybrid Systems 32 (2019) 294-305.

[25] X. Yang, X. Li, Q. Xi, P. Duan, Review of stability and stabilization for impulsive delayed systems, Mathematical Biosciences and Engineering 15 (6) (2018) 1495-1515.

[26] X. Li, X. Yang, T. Huang, Persistence of delayed cooperative models: Impulsive control method, Applied Mathematics and Computation 342 (2019) 130-146.

[27] W.-H. Chen, G. Yuan, W. X. Zheng, Robust stability of singularly perturbed impulsive systems under nonlinear perturbation, IEEE Transactions on Automatic Control 58 (1) (2013) 168-174.

[28] W.-H. Chen, D. Wei, X. Lu, Exponential stability of a class of nonlinear singularly perturbed systems with delayed impulses, Journal of the Franklin Institute 350 (9) (2013) 2678-2709.

[29] W. K. Wong, W. Zhang, Y. Tang, X. Wu, Stochastic synchronization of complex networks with mixed impulses, IEEE Transactions on Circuits and Systems I: Regular Papers 60 (10) (2013) 2657-2667.

[30] N. Wang, X. Li, J. Lu, F. E. Alsaadi, Unified synchronization criteria in an array of coupled neural networks with hybrid impulses, Neural Networks 101 (2018) 25-32.

[31] Y. Wang, J. Lu, J. Liang, J. Cao, M. Perc, Pinning synchronization of nonlinear coupled Lur' e networks under hybrid impulses, IEEE Transactions on Circuits and Systems II: Express Briefs 66 (3) (2018) $432-436$.

[32] W. Zhang, Y. Tang, X. Wu, J.-A. Fang, Synchronization of nonlinear dynamical networks with heterogeneous impulses, IEEE Transactions on Circuits and Systems I: Regular Papers 61 (4) (2013) 1220-1228.

[33] Y. Tang, H. Gao, W. Zhang, J. Kurths, Leader-following consensus of a class of stochastic delayed multi-agent systems with partial mixed impulses, Automatica 53 (2015) 346-354. 